\title{
ELIMINATION OF SCAN IMPEDANCE ANOMALIES IN ULTRA-WIDE BAND PHASED ARRAYS OF DIFFERENTIALLY FED TAPERED SLOT ANTENNA ELEMENTS
}

\author{
E. García1, E. de Lera2, D. Segovia1, V. González3, J. A. López-Fernández4 \\ ${ }^{1}$ Dpto. Teoría de Señal y Comunicaciones. Universidad Carlos III de Madrid. Spain \\ legarcia@tsc.uc3m.es \\ ${ }^{2}$ Astrophysics Group, Cavendish Laboratory. University of Cambridge. United Kingdom. \\ eloy@mrao.cam.ac.uk \\ ${ }^{3}$ Dpto. de Ingeniería Audiovisual y Comunicaciones. Universidad Politécnica de Madrid. \\ vgonzalz@diac.upm.es \\ ${ }^{4}$ Centro Astronómico de Yebes. Observatorio Astronómico Nacional. Spain. \\ ja.lopez@oan.es
}

\begin{abstract}
Scan impedance anomalies are associated to phased arrays. There are different phenomena in order to explain these scan blindnesses produced when the array scans at one frequency and one angle. The most popular technique for eliminating these scan blindnesses is based on the use of electric walls in the grid of the two dimensional array. In this paper we present a dual polarized ultra-wide band array based on "bunny ears" antennas from $0.3 \mathrm{GHz}$ to $1 \mathrm{GHz}$ which presents scan blindness. We analyze the cause of the anomaly and present a technique non based on electric walls in order to avoid the scan blindness with a small increase in the noise figure of the array.
\end{abstract}

\section{INTRODUCTION}

Scan blindnesses are a problem inherent to phased arrays antennas with strong mutual coupling between elements. Important efforts have been done by several authors not only for eliminating these anomalies but also for giving and explanation to the phenomena $[1,2,3]$. In this paper we present an ultra-wide band array based on a particular kind of Vivaldi antenna called "bunny ears" antennas. The frequency work band is from $0.3 \mathrm{GHz}$ to $1 \mathrm{GHz}$. The array presents a dual polarization and it has been designed following the specifications of the Square Kilometer Array (SKA) [3] for application as a radio astronomy receiver. We present an explanation for the scan blindness observed in this array based on the studies of Schaubert [1] and also we present a technique for eliminating the impedance anomaly without using electric walls or by using the orthogonal antenna element as an electric wall. Our technique is based on canceling the even mode induced currents in the structure due to the parallel plate mode propagating through the structure. We associate these induced currents to the principal cause of the mismatching impedance which produces the scan blindness phenomena. Finally a noise analysis is performed yielding very satisfactory results. All simulations presented here have been done with HFSS v.10 software for an infinite array system.

\section{SCAN BLINDNESS DISCUSSION}

First we present the phased array geometry for a better understanding of the scan blindness. In Figure 1 we can see the geometry of the array and the basic radiating element. The array is based on a rectangular lattice of "bunny ears" antennas. The frequency band is from $0.3 \mathrm{GHz}$ to $1 \mathrm{GHz}$. The operation band could be bigger but this is the specification of the SKA project in which this work is framed. The array works at dual polarization scanning from $-45^{\circ}$ to $45^{\circ}$. In order to get minimum fabrication cost and simplicity the antenna is not printed on a substrate. The array is designed using aluminum sheets. Other important advantage of this array is that is based on differential technology. The antenna is connected to the amplifier avoiding the use of a noisy balun. 
In Figure 2, we present the VSWR of an infinite by infinite array with a ground plane. It is necessary to use a ground plane because the amplifier stage and the beam forming network (BFN) are placed after the antenna layer. These two RF stages require a common ground plane. Furthermore, the earth at these frequencies could be a ground plane, and a ground plane would also be necessary in this solution for giving more mechanical stability to the array. In Figure 2 we can see marked with an arrow the scan blindness typically found in the E plane scan. Following Hansen and Schaubert explanations, we could find several origins for these anomalies. [1, 2, 4]. We discarded the origin of the scan blindness in the array due to surface waves, because we do not have substrate that could support that kind of waves. Also we discarded the origin of the anomalies due to gaps between antennas as far as they are completely connected in every row. According to Schaubert studies [5] we could think in the possibility of radiation due to the feed lines of the antennas. However, our simulations do not agree with these kinds of anomalies. Increasing the scan angle the frequency of the anomaly decreased which is the phenomenon opposite to that explained by Schaubert in [5]. Other explanation could be linked to the presence of parallel plate modes due to the corrugated geometry of the array, as Schaubert explained $[1,2]$. Simulations of the same array with one polarization show the same anomalies explained by the model of Schaubert. We want to go beyond the elegant Schaubert studies in a simpler manner. With no ground plane and double polarization, simulations of the array do not produce scan blindness. Using a ground plane in the bottom of the array we observe the scan blindness. We could think that in the first case we have two conductors (the two arms of the antenna) therefore the system is balanced as a transmission line. Introducing a ground plane is similar to introduce a third conductor, so this structure could support an even mode induced by the parallel plate mode propagating through the structure. These induced currents which generate the even mode in the antenna arms are the responsible of the scan blindness. Our idea is to eliminate this even mode. Placing an electric wall instead of the second orthogonal antenna elements in the lattice the scan blindness disappear, as Schaubert shown [1, 2], losing the dual polarization capability. We present here another technique using two resistors per element. Each resistor connects one of the conductors of the feed line to the GND plane. Even if this solution may seem very noisy for a radio astronomy application, analyzing the noise figure of the array, we realize that the noise increment is not a big drawback. This solution is similar to a balanced structure.In Figure 3 we present the results using $200 \Omega$ resistors. This value is very close to the input scan impedance of the antenna when the array is pointing at broadside at the sampling frequency $(0.857 \mathrm{GHz})$. We see that the scan blindness disappears. A parametric study for the resistor values was done. The conclusion is that for $1 \Omega$ resistor, the scan blindness is still present (this situation is similar to short-circuiting the arms of the antenna to the ground plane). Increasing the resistor tens of ohms the scan blindness quickly disappears. When the resistor is $10 \mathrm{k} \Omega$ no scan blindness phenomena is observed but the system is strongly mismatched. In Figure 4 we present the VSWR in an E-plane scan for the full frequency band and for three scanning angles for $1 \mathrm{k} \Omega$ and $3 \mathrm{k} \Omega$ resistors. The H-plane scan response is not affected by the resistors. We can see that with $3 \mathrm{k} \Omega$, the matching level in the band remains acceptable even in high scanning angles and the results are very similar to those obtained from a resistor value of $1 \mathrm{k} \Omega$. At this point it is necessary a noise study of the solution.

\section{NOISE STUDY OF THE SOLUTION PROPOSED}

We present here our results of the noise figure increasing with respect to the array without resistors. The analysis is done taking into account the resistor value. We use a real resistor SMD 1206 for simulating the antenna noise with Microwave Office 
software. We have noticed in our simulations that increasing the resistor value the noise figure decreases and the impedance matching degrades. In Figure 5 the results for two values of resistor are presented. Resistors of $1 \mathrm{k} \Omega$ and $3 \mathrm{k} \Omega$ are used for all the frequency values of the operating band and for the complete scanning angle margin. The conclusion is that it is necessary a compromise solution increasing the resistor value in order to obtain a lower noise figure but with a good impedance matching. A possible solution is to choose a resistor value of $3 \mathrm{k} \Omega$ which implies good values of noise (Figure 5) and a good matching level as seen in Figure 4.

\section{CONCLUSIONS}

We present in this paper an ultra-wide band array for radio astronomy from 0.3 $\mathrm{GHz}$ to $1 \mathrm{GHz}$ with dual polarization based on aluminum sheet "bunny ears" antennas. These kinds of antennas give a lot of important advantages such as the use of differential technology and the reduction of the cost of the system. The phased array presents scan blindness in E plane scans. We present here a technique for avoiding that problem based on the use of resistors. The resistors are used to cancel the even mode currents induced in the antennas due to the parallel plate mode propagating through the structure. A study of the noise figure of the array is also presented and good results are achieved. A parameter study of different values of the resistors is done, pointing to a compromise solution between low noise for high resistor values and low mismatch for low resistor values.

\section{REFERENCES}

[1] Schaubert, D. "A class of E-plane Scan Blindnesses in single-polarized arrays of tapered-slot antennas with a ground plane", IEEE Transactions on Antennas and Propagation, July 1996, Vol. 44, nº 7, pp. 954-959.

[2] Wunsch J, Schaubert D., "Full and partial crosswalls between unit cells of endfire slotline arrays", IEEE Transactions on Antennas and Propagation, June 2000, Vol. 48, nº, pp. 981-986.

[3] www.skatelescope.org

[4] R. C. Hansen, "Phased Arrays Antennas", John Wiley \& Sons Inc., 2001.

[5] Schaubert, D. "E-plane scan performance of infinite arrays of dipoles on protruding dielectric substrates: coplanar feed line and E-plane metallic wall effects", IEEE Transactions on Antennas and Propagation, June 1993, Vol. 41, nº, pp. 837841.

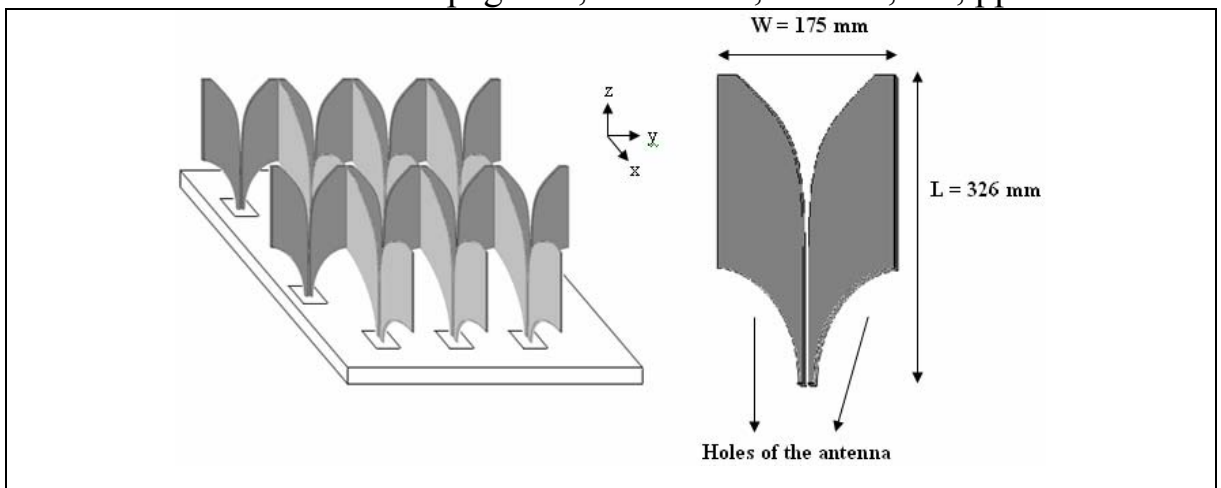

Figure 1: Geometry of a portion of the infinite by infinite array with ground plane. Each polarization in a different color (left). Antenna element (right). 


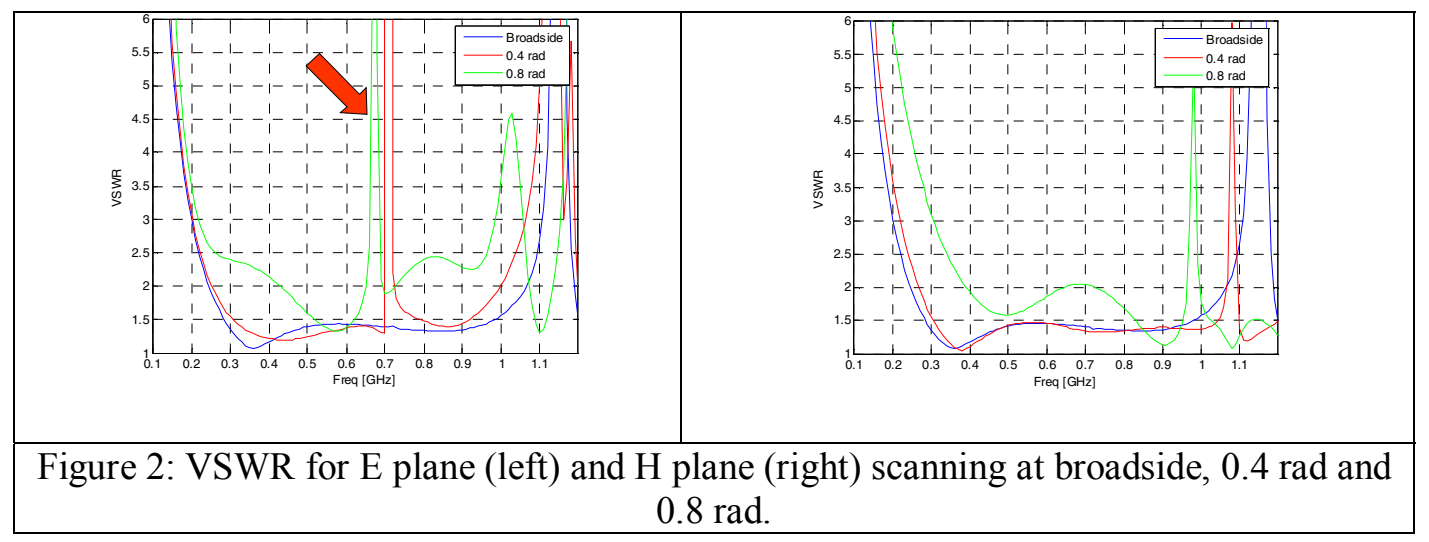

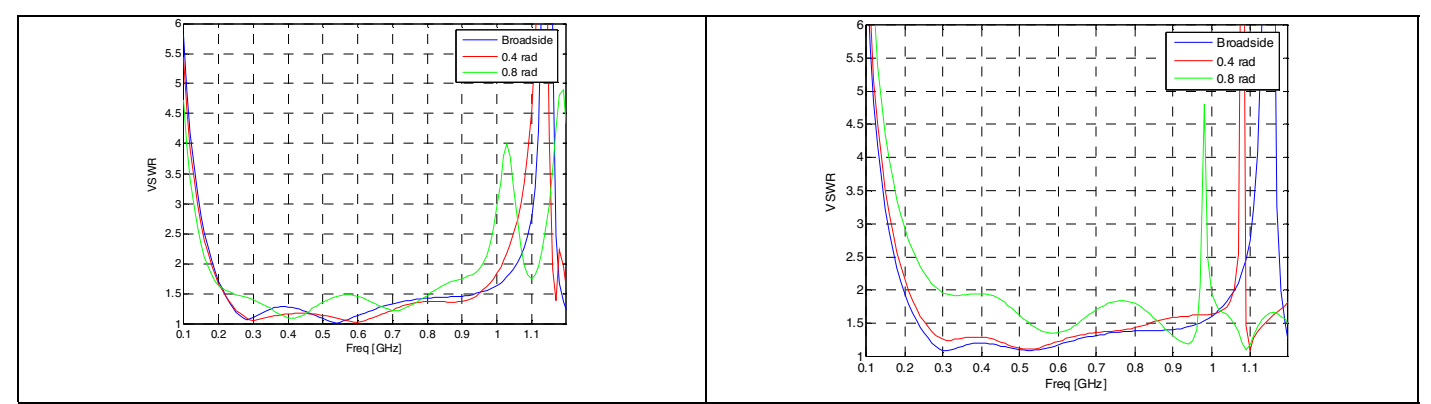

Figure 3: VSWR for E plane (left) and H plane (right) scanning at broadside, 0.4 rad and $0.8 \mathrm{rad}$ with $200 \Omega$ resistors in each arm of the antenna.

\begin{tabular}{|c|c|c|}
\hline & \\
\hline
\end{tabular}
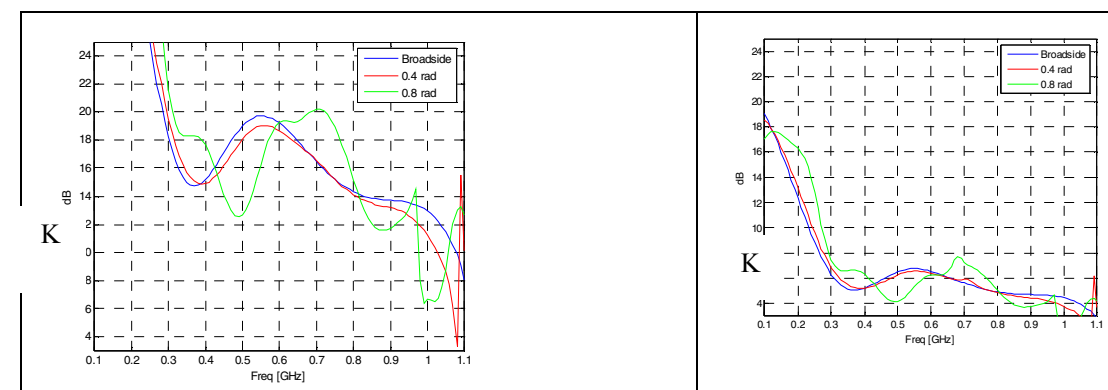

Figure 5: Noise temperature increasing in $\mathrm{K}$ for $1 \mathrm{k} \Omega$ (left) and $3 \mathrm{k} \Omega$ (right) resistors in the E plane scan. 